\title{
Plasma Dihydroxyphenylglycol and the Intraneuronal Disposition of Norepinephrine in Humans
}

\author{
David S. Goldstein," Graeme Eisenhofer," Robin Stull," Carol Joan Folio,“ Harry R. Keiser," and Irwin J. Kopin” \\ ${ }^{*}$ Hypertension-Endocrine Branch, National Heart, Lung, and Blood Institute, ${ }^{\ddagger}$ Intramural Research Program, National Institute of \\ Neurological and Communicative Disorders and Stroke, Bethesda, Maryland 20892
}

\begin{abstract}
We examined plasma levels of the sympathetic neurotransmitter norepinephrine (NE) and its deaminated metabolite dihydroxyphenylglycol (DHPG) during supine rest in healthy human subjects and in sympathectomized patients, during physiological (tilt) or pharmacological (yohimbine, clonidine) manipulations known to affect sympathetically mediated NE release, during blockade of neuronal uptake of NE (uptake-1) using desipramine, and during intravenous infusion of $\mathrm{NE}$. Healthy subjects had a mean arteriovenous increment in plasma DHPG in the arm $(10 \%, P<0.05)$, whereas sympathectomized patients had a mean arteriovenous decrement in DHPG in the affected limb (mean decrease $21 \%, P<0.05$ compared with healthy subjects). Tilt and yohimbine, which stimulate, and clonidine, which inhibits, release of endogenous NE, produced highly correlated changes in plasma NE and DHPG $(r=0.94)$. Pretreatment with desipramine abolished DHPG responses to yohimbine while enhancing NE responses. To attain a given increase in plasma DHPG, about a tenfold larger increment in arterial NE was required during NE infusion than during release of endogenous NE. When plasma NE was markedly suppressed after administration of clonidine, plasma DHPG decreased to a plateau level of $700-800 \mathrm{pg} / \mathrm{ml}$. The results indicate that (i) plasma DHPG in humans is derived mainly from sympathetic nerves; (ii) increments in plasma DHPG during stimulation of $\mathrm{NE}$ release result from uptake of NE into sympathetic nerve endings and subsequent intraneuronal conversion to DHPG; (iii) plasma DHPG under basal conditions probably is determined mainly by net leakage of $\mathrm{NE}$ into the axonal cytoplasm from storage vesicles; and (iv) increments in NE concentrations at neuronal uptake sites can be estimated by simultaneous measurements of DHPG and NE during NE infusion and NE release. Measurement of NE and DHPG provides unique clinical information about sympathetic function.
\end{abstract}

\section{Introduction}

Dihydroxyphenylglycol (DHPG) ${ }^{1}$ is a deaminated metabolite of the sympathetic neurotransmitter norepinephrine (NE).

Address correspondence to Dr. D. S. Goldstein, Bldg. 10 7N238, NHLBI, NIH, Bethesda, MD 20892.

Received for publication 29 January 1987 and in revised form 16 July 1987.

1. Abbreviations used in this paper: DA, dopamine; DHPG, dihydroxyphenylglycol; DMI, desipramine; MAO, monoamine oxidase; $\mathrm{NE}$, norepinephrine; $\mathrm{YOH}$, yohimbine.

The Journal of Clinical Investigation, Inc.

Volume 81, January 1988, 213-220
Results of in vitro studies and studies of laboratory animals have led to the conclusion that DHPG is a prominent intraneuronal metabolite of NE and that DHPG formed in isolated tissues is derived predominantly from neuronal as opposed to nonneuronal sources (see reference 1 for review).

DHPG is present in plasma of humans $(2,3)$ at concentrations that generally are severalfold higher than those of NE. The source and meaning of DHPG in human plasma, however, are poorly understood. In the present study we examined how plasma DHPG reflects the rate and site of NE metabolism.

Plasma levels of the sympathetic neurotransmitter, NE, often have been used in clinical studies of cardiovascular and neuropsychiatric disorders as an index of sympathetic nervous system activity (4). Plasma levels of NE are determined, however, not only by sympathetic nerve traffic but also by intraneuronal NE synthesis, presynaptic modulation of transmitter release into the synaptic clefts, neuronal reuptake (uptake-1), extraneuronal uptake and metabolism, the site of sampling, diffusion, and excretion. The limitations of measuring plasma $\mathrm{NE}$ alone to indicate sympathetic function are by now widely recognized.

In the present study, a recently developed liquid chromatographic-electrochemical assay technique for measuring simultaneously DHPG and catecholamines (2) was used to establish in humans whether plasma DHPG can reflect intraneuronal disposition of NE and provide information about sympathetic function unobtainable by measuring plasma NE alone.

\section{Experimental approach}

The experimental approach we used can most easily be understood by considering processes depicted in Fig. 1.

Catecholamine biosynthesis begins with uptake of the amino acid tyrosine into the sympathetic neuronal cytoplasm and conversion of tyrosine to dihydroxyphenylalanine by tyrosine hydroxylase and then to dopamine (DA) by L-amino acid-decarboxylase (5). DA in the cytoplasm is taken up into vesicles containing DA-beta-hydroxylase and converted to $\mathrm{NE}$. NE in the vesicle can be stored, can leak back into the cytoplasm, or can be released into the synapse. The uptake of cytoplasmic NE or DA into the vesicle can be blocked by reserpine. According to generally accepted concepts about noradrenergic neurotransmission, sympathetic stimulation causes exocytotic release of the soluble contents of the vesicle, including DA-beta-hydroxylase and NE, into the synaptic cleft. The released NE can bind to post- or extrasynaptic receptors to elicit physiological responses; can bind to presynaptic alpha-2 adrenoceptors to diminish NE release for a given amount of sympathetic nerve traffic; can be taken back up into the axonal cytoplasm via uptake-1; or can be taken up into extraneuronal cells such as smooth muscle or endothelial cells. Yohimbine (YOH) is an alpha-2 adrenoceptor inhibitor, and desipramine (DMI) inhibits neuronal uptake of NE (uptake-1). NE in 


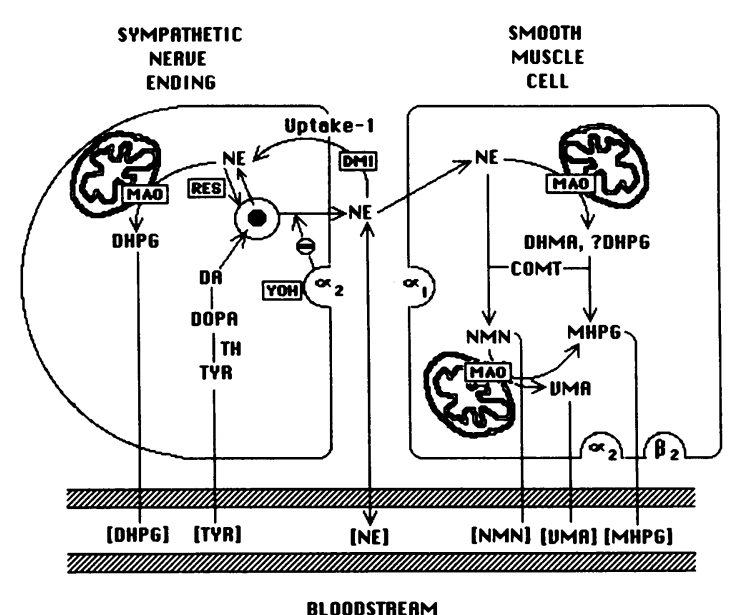

Figure 1. Diagram of events at sympathetic neuroeffector junction. TYR, tyrosine; DOPA; dihydroxyphenylalanine; TH, hydroxylase; RES, reserpine; COMT, catechol-O-methyltransferase; NMN, normetanephrine; MHPG, 3-methoxy-4-hydroxyphenylglycol; VMA, vanillylmandelic acid; DHMA, dihydroxymandelic acid.

extraneuronal cells can be metabolized by catechol- $O$-methyltransferase to form normetanephrine, or, after deamination of normetanephrine catalyzed by monoamine oxidase (MAO), to form 3-methoxy-4-hydroxyphenylglycol or vanillylmandelic acid, end products of NE metabolism. Relative amounts of dihydroxymandelic acid and DHPG formed extraneuronally are unknown.

\section{Fate of cytoplasmic NE}

$\mathrm{NE}$ is removed rapidly from the axonal cytoplasm by uptake into storage vesicles and by deamination by MAO, which is located in the outer mitochondrial membrane in both neuronal and extraneuronal cells. The resulting aldehyde can be reduced, to form DHPG, or oxidized to dihydroxymandelic acid. Results of in vitro studies including effects of MAO inhibition have indicated that the main deaminated metabolite of NE in sympathetic neural tissue is DHPG (1). Unlike NE, DHPG can easily traverse the cell membrane and diffuse into the general circulation.

\section{Predicted effects of physiological or pharmacological manipulations}

This framework, based mainly on in vitro studies or studies of isolated, perfused organs, provided the basis for several hypotheses about plasma DHPG.

Stimuli that release endogenous NE, such as tilt or administration of the alpha-2 adrenoceptor antagonist, YOH, would be expected to increase plasma DHPG, whereas administration of the alpha- 2 adrenocepter agonist, clonidine, would be expected to decrease plasma DHPG, because these manipulations would affect the amount of $\mathrm{NE}$ available for reuptake by uptake-1 and subsequent intraneuronal metabolism.

If $\mathrm{NE}$ recaptured after release were the main source of increases in plasma DHPG during sympathetic stimulation, then blockade of neuronal uptake would block stimulation-induced increments in plasma DHPG. Thus, in subjects pretreated with DMI, which inhibits uptake of NE into sympathetic nerve terminals, increments in plasma NE produced by
YOH administration would not be expected to be attended by increases in plasma DHPG. During intravenous infusion of NE, plasma DHPG also would be expected to increase, but the increment would be smaller than during release of similar amounts of endogenous NE because of the large plasma-synaptic cleft concentration gradient for NE. This increase would also be attenuated in subjects pretreated with DMI.

If basal release of DHPG into the circulation depended on reuptake of NE spontaneously released into the synaptic clefts, then administration of DMI or clonidine would decrease plasma DHPG to low levels; however, if plasma DHPG at baseline derived from leakage of NE from intraneuronal storage sites, then treatment with DMI or clonidine would have only minor effects on plasma DHPG; substantial plasma levels of DHPG still would be observed.

\section{Methods}

\section{Subjects}

The clinical data base was derived from assays of plasma samples from subjects who had previously participated in studies involving plasma catecholamine pharmacokinetics or responses to stimuli known to affect sympathetically mediated release of $\mathrm{NE}$ into the circulation $(6-10)$. All human subjects consented in writing to participate in the protocols, which were approved by Institutional Review Boards of the National Institutes of Health (NIH). The results of the measurements of plasma DHPG are reported here for the first time. The subjects all were either normotensive volunteers between 18- and 55-yr old who were recruited through the NIH Normal Volunteer Program, or else patients of similar age who were healthy except for mild essential hypertension. All patients had discontinued antihypertensive medications for at least 2 wk before study.

\section{Conditions of testing and blood sampling}

Intravenously infused drugs were administered into an antecubital vein, with blood sampled from the opposite arm.

Blood was sampled through indwelling catheters into chilled, heparinized, evacuated glass tubes without other additives, the plasma separated by refrigerated centrifugation, and the samples stored at $-70^{\circ} \mathrm{C}$ until the time of assay within $\sim 4 \mathrm{wk}$. Under these conditions, we have observed no trends in DHPG levels in aliquots of standard plasma over storage periods of up to several months.

\section{Normal values at supine rest}

Arterial and venous plasma was obtained from 19 subjects after they had been at rest supine for at least $20 \mathrm{~min}$. In each subject, a 20-gauge, 2-in-long Teflon catheter was inserted percutaneously after infiltration of the overlying skin using lidocaine. Dilute heparin was flushed manually as needed into the catheter using a system of tubing and stopcocks that was closed to air. Brachial arterial blood pressure was recorded continuously using a Statham P23De transducer and Gould eight channel polygraph.

Also, arterial and venous blood samples were obtained from four patients who had undergone a regional sympathectomy for causalgia.

\section{Experimental manipulations}

YOH

Six normotensive subjects, five men and one woman, age 23-27-yr old, received a bolus intravenous injection of $0.125 \mathrm{mg} / \mathrm{kg} \mathrm{YOH}$ followed by an intravenous infusion of $\mathrm{YOH}$ at $0.001 \mathrm{mg} / \mathrm{kg}$ per min. Arterial and antecubital venous blood was sampled at 15 min during the infusion. Five other normotensive men, age range 22-23-yr old, were pretreated with $125 \mathrm{mg}$ oral DMI $\sim 2 \mathrm{~h}$ before YOH administration. 
TILT

Five normotensive men, age 22-34-yr old, had arterial and venous blood drawn while they were supine and after at least 5 min each at 30 , 45 , and 60 degrees from horizontal using a tilt table.

NE

Eight normotensive men, age 22-52-yr old, received an intravenous infusion of NE, $5 \mathrm{mg}$ base/liter 5\% dextrose, at rates titrated to increase mean arterial pressure by 10,20 , and $30 \mathrm{~mm} \mathrm{Hg}$ in random order. After at least $10 \mathrm{~min}$ at each infusion rate, arterial and venous blood was sampled. Three other normotensive men, age 22-23-yr old, were pretreated with 125 oral DMI $\sim 2 \mathrm{~h}$ before $\mathrm{YOH}$ administration.

\section{CLONIDINE}

Eight subjects, two hypertensive and six normotensive, six men and two women, age 22-38-yr old, had supine antecubital venous blood samples drawn via an indwelling catheter or needle at baseline and at 1 , 2,3 , and $3.5 \mathrm{~h}$ after oral administration of $300 \mu \mathrm{g}$ clonidine.

\section{DMI}

Five normotensive men, age 22-24-yr old, had supine antecubital venous samples drawn via an indwelling catheter or needle at baseline and at 1,2 , and $6 \mathrm{~h}$ after oral administration of $125 \mathrm{mg} \mathrm{DMI}$.

\section{Assay procedures}

To conduct the studies reported here, we developed and validated a liquid chromatographic-electrochemical method for quantifying simultaneously plasma levels of DHPG, the catecholamine precursor dihydroxyphenylalanine, and the catecholamines. The method was described in detail previously (2). Briefly, after addition of an internal standard (dihydroxybenzylamine) to the plasma, the catechols were extracted by absorption on alumina. After elution from the alumina, they were separated by reversed-phase, ion-pairing liquid chromatography and quantified by the current produced upon exposure of the column effluent to a series of oxidizing electrodes and then a reducing electrode. The limit of detection was $\sim 15 \mathrm{pg} / \mathrm{ml}$ for DHPG and $\sim 10$ $\mathrm{pg} / \mathrm{ml}$ for the catecholamines. Intra- and interassay coefficients of variation were $5.9 \%$ and $10.1 \%$, respectively, at mean plasma DHPG concentrations of 992 and $924 \mathrm{pg} / \mathrm{ml}$. The correlation for the scatter plot relating calculated plasma DHPG and DHPG added as an external standard was 0.99 . The recovery of DHPG averaged $71 \%$ of the recovery of the internal standard, dihydroxybenzylamine, whereas the recovery of NE averaged $99 \%$ of the recovery of the internal standard. Concentrations of DHPG were adjusted for this difference by using the recovery of DHPG in an extracted mixture of standards during each assay of samples.

\section{Data analysis}

Statistical analyses included dependent- and independent-means $t$ tests and linear regression. A $\boldsymbol{P}$ value $<0.05$ defined statistical significance. Mean values were expressed \pm SEM.

\section{Results}

\section{Values at supine rest}

Average values for arterial and venous DHPG and NE are displayed in Tables I and II and Fig. 2. Antecubital venous DHPG exceeded arterial DHPG in most normal subjects, and there was a small but statistically significant mean arteriovenous increment in plasma DHPG $(10 \%, t=2.08, P<0.05)$. Arterial DHPG exceeded venous DHPG in three patients with regional sympathectomies (mean arteriovenous decrement $21 \%, t=2.03, P<0.05$ compared with subjects with intact sympathetic innervation). In one sympathectomized patient, data were obtained only for plasma NE and not DHPG.
Table I. Plasma DHPG Concentrations in Humans

\begin{tabular}{|c|c|c|}
\hline Condition & Arterial & Venous \\
\hline & $p g / m l$ & $p g / m l$ \\
\hline Controls (19) & $716 \pm 56^{*}$ & $789 \pm 56$ \\
\hline Sympathectomized (3) & $601 \pm 121$ & $476 \pm 111$ \\
\hline \multicolumn{3}{|l|}{ YOH (6) } \\
\hline Baseline & $854 \pm 82$ & $896 \pm 105$ \\
\hline YOH & $1,040 \pm 91$ & $1,128 \pm 160$ \\
\hline \multicolumn{3}{|l|}{ YOH + DMI (5) } \\
\hline Baseline & $487 \pm 35$ & $503 \pm 41$ \\
\hline YOH & $483 \pm 38$ & $509 \pm 38$ \\
\hline \multicolumn{3}{|l|}{ NE Infusion (8) } \\
\hline Baseline & $657 \pm 73$ & $745 \pm 100$ \\
\hline$\Delta=10 \mathrm{~mm} \mathrm{Hg}$ & $710 \pm 74$ & $769 \pm 104$ \\
\hline$\Delta=20 \mathrm{~mm} \mathrm{Hg}$ & $760 \pm 93$ & $778 \pm 97$ \\
\hline$\Delta=30 \mathrm{~mm} \mathrm{Hg}$ & $967 \pm 115$ & $936 \pm 100$ \\
\hline \multicolumn{3}{|l|}{ NE Infusion, DMI (3) } \\
\hline Baseline & $508 \pm 39$ & $554 \pm 54$ \\
\hline$\Delta=10 \mathrm{~mm} \mathrm{Hg}$ & $505 \pm 66$ & $486 \pm 49$ \\
\hline$\Delta=20 \mathrm{~mm} \mathrm{Hg}$ & $479 \pm 68$ & $558 \pm 75$ \\
\hline$\Delta=\mathbf{3 0} \mathrm{mm} \mathrm{Hg}$ & $501 \pm 27$ & $579 \pm 58$ \\
\hline \multicolumn{3}{|l|}{ Tilt (5) } \\
\hline Supine & $732 \pm 88$ & $846 \pm 109$ \\
\hline $30^{\circ}$ & $780 \pm 80$ & $827 \pm 85$ \\
\hline $45^{\circ}$ & $836 \pm 81$ & $858 \pm 80$ \\
\hline $60^{\circ}$ & $883 \pm 53$ & $932 \pm 82$ \\
\hline \multicolumn{3}{|l|}{ Clonidine (11) } \\
\hline Baseline & & $883 \pm 66$ \\
\hline $1 \mathrm{~h}$ & & $818 \pm 67$ \\
\hline $2 \mathrm{~h}$ & & $770 \pm 54$ \\
\hline $3 \mathrm{~h}$ & & $770 \pm 66$ \\
\hline $3.5 \mathrm{~h}$ & & $773 \pm 71$ \\
\hline \multicolumn{3}{|l|}{ DMI (5) } \\
\hline Baseline & & $969 \pm 84$ \\
\hline $1 \mathrm{~h}$ & & $1,018 \pm 103$ \\
\hline $2 \mathrm{~h}$ & & $871 \pm 80$ \\
\hline $6 \mathrm{~h}$ & & $788 \pm 94$ \\
\hline
\end{tabular}

Numbers in parentheses represent numbers of subjects.

* Mean values \pm SEM.

\section{YOH}

Intravenously administered YOH increased plasma DHPG in all subjects tested (mean increase $22 \%$ arterial, $26 \%$ venous, $t$ $=3.86$ and $t=3.50, P<0.01$ ). In contrast, YOH had no effect on arterial or venous DHPG in subjects pretreated with DMI (Fig. 2).

\section{$N E$ infusion}

Intravenous administration of $\mathrm{NE}$ caused dose-related increases in plasma DHPG (Table I, and Fig. 3). The increases in plasma DHPG, although statistically significant, were small compared with the increases in plasma NE. For instance, at a $\mathrm{NE}$ infusion rate that produced a $30-\mathrm{mm} \mathrm{Hg}$ pressor response, arterial $\mathrm{NE}$ averaged $\sim 6,200 \mathrm{pg} / \mathrm{ml}$ ( a 40-fold increase from baseline), whereas arterial DHPG averaged $\sim 1,000$ $\mathrm{pg} / \mathrm{ml}$ ( $\sim$ a 1.5-fold increase from baseline), $t=3.20, P<0.01$ for arterial DHPG). In subjects pretreated with DMI, plasma 
Table II. Plasma NE Concentrations in Humans

\begin{tabular}{|c|c|c|}
\hline Condition & Arterial & Venous \\
\hline & $p g / m l$ & $p g / m l$ \\
\hline Controls (19) & $169 \pm 19^{*}$ & $202 \pm 21$ \\
\hline Sympathectomized (4) & $251 \pm 44$ & $171 \pm 19$ \\
\hline \multicolumn{3}{|l|}{ YOH (6) } \\
\hline Baseline & $175 \pm 10$ & $245 \pm 28$ \\
\hline YOH & $329 \pm 40$ & $473 \pm 92$ \\
\hline \multicolumn{3}{|l|}{ YOH + DMI (5) } \\
\hline Baseline & $121 \pm 8$ & $167 \pm 17$ \\
\hline YOH & $470 \pm 113$ & $1,414 \pm 735$ \\
\hline \multicolumn{3}{|l|}{ NE Infusion (8) } \\
\hline Baseline & $150 \pm 20$ & $169 \pm 21$ \\
\hline$\Delta=10 \mathrm{~mm} \mathrm{Hg}$ & $1,614 \pm 307$ & $808 \pm 155$ \\
\hline$\Delta=20 \mathrm{~mm} \mathrm{Hg}$ & $3,848 \pm 837$ & $1,659 \pm 274$ \\
\hline$\Delta=30 \mathrm{~mm} \mathrm{Hg}$ & $6,197 \pm 1,144$ & $2,925 \pm 638$ \\
\hline \multicolumn{3}{|l|}{ NE Infusion, DMI (3) } \\
\hline Baseline & $118 \pm 6$ & $164 \pm 26$ \\
\hline$\Delta=10 \mathrm{~mm} \mathrm{Hg}$ & $294 \pm 58$ & $249 \pm 5$ \\
\hline$\Delta=20 \mathrm{~mm} \mathrm{Hg}$ & $418 \pm 49$ & $448 \pm 103$ \\
\hline$\Delta=30 \mathrm{~mm} \mathrm{Hg}$ & $972 \pm 169$ & $533 \pm 50$ \\
\hline \multicolumn{3}{|l|}{ Tilt (5) } \\
\hline Supine & $124 \pm 18$ & $177 \pm 29$ \\
\hline $30^{\circ}$ & $194 \pm 27$ & $236 \pm 35$ \\
\hline $45^{\circ}$ & $253 \pm 40$ & $294 \pm 43$ \\
\hline $60^{\circ}$ & $283 \pm 47$ & $357 \pm 57$ \\
\hline \multicolumn{3}{|l|}{ Clonidine (11) } \\
\hline Baseline & & $159 \pm 54$ \\
\hline $1 \mathrm{~h}$ & & $95 \pm 46$ \\
\hline $2 \mathrm{~h}$ & & $61 \pm 38$ \\
\hline $3 \mathrm{~h}$ & & $55 \pm 29$ \\
\hline $3.5 \mathrm{~h}$ & & $57 \pm 37$ \\
\hline \multicolumn{3}{|l|}{ DMI (5) } \\
\hline Baseline & & $156 \pm 13$ \\
\hline $1 \mathrm{~h}$ & & $191 \pm 22$ \\
\hline $2 \mathrm{~h}$ & & $174 \pm 15$ \\
\hline $6 \mathrm{~h}$ & & $211 \pm 54$ \\
\hline
\end{tabular}

Numbers in parentheses represent numbers of subjects.

* Mean values \pm SEM.

NE concentrations associated with given pressor responses were markedly smaller than those for the same pressor responses in untreated subjects (Table II). Subjects pretreated with DMI had no change in plasma DHPG during intravenous infusion of NE (Table I).

\section{Clonidine}

Oral administration of clonidine decreased plasma DHPG significantly (Table I). The decreases in plasma DHPG occurred over about the same time course as decreases in plasma NE (Table II) and were statistically significant by $2 \mathrm{~h}$ after drug administration $(t=4.00, P<0.01)$.

In contrast with the results for NE infusion, during stimuli affecting release of endogenous $\mathrm{NE}$ (tilt, $\mathrm{YOH}$, clonidine), the mean change in plasma NE was associated with a similar mean change in plasma DHPG (Fig. 4). As indicated in Fig. 5, plasma DHPG generally paralleled plasma NE during stimuli affecting release of NE. When plasma NE was markedly sup-

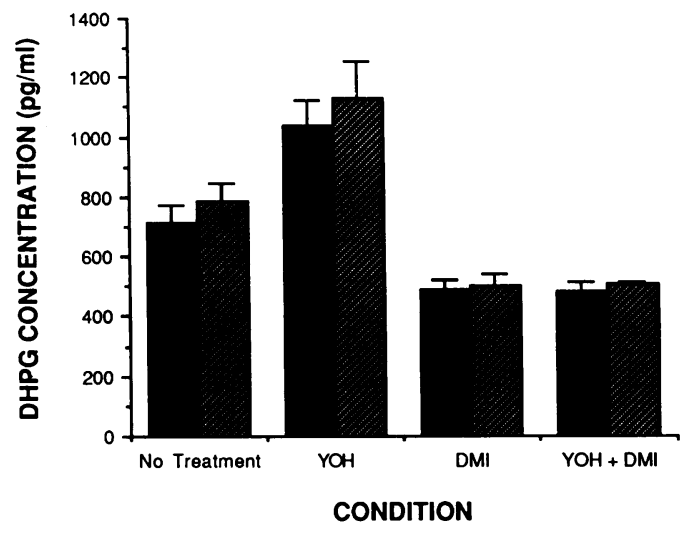

Figure 2. Arterial and venous mean levels of DHPG (DHPGa [匹] and DHPGv [ $\left.\left.{ }^{\circ}\right]\right)$ at baseline, during administration of $\mathrm{YOH}$, at baseline after administration of oral DMI, and during administration of $\mathrm{YOH}$ in subjects pretreated with DMI (YOH + DMI). A small arteriovenous increment in DHPG was observed at baseline. YOH increased DHPGa and DHPGv. Pretreatment with DMI abolished these increases.

pressed after clonidine administration, plasma DHPG decreased to a value between 700 and $800 \mathrm{pg} / \mathrm{ml}$.

\section{$D M I$}

Oral administration of DMI caused small decreases in plasma DHPG that were not statistically significant until the 6-h sampling point $(-19 \%, t=4.47, P<0.01)$.

\section{Discussion}

\section{Source and meaning of plasma DHPG during altered} release of endogenous $N E$

The present results indicate that plasma levels of DHPG are affected by manipulations that enhance or inhibit release of NE from sympathetic nerve terminals or that inhibit uptake of NE into nerve terminals. Similar absolute increases in plasma DHPG and NE were observed during tilt or intravenous ad-

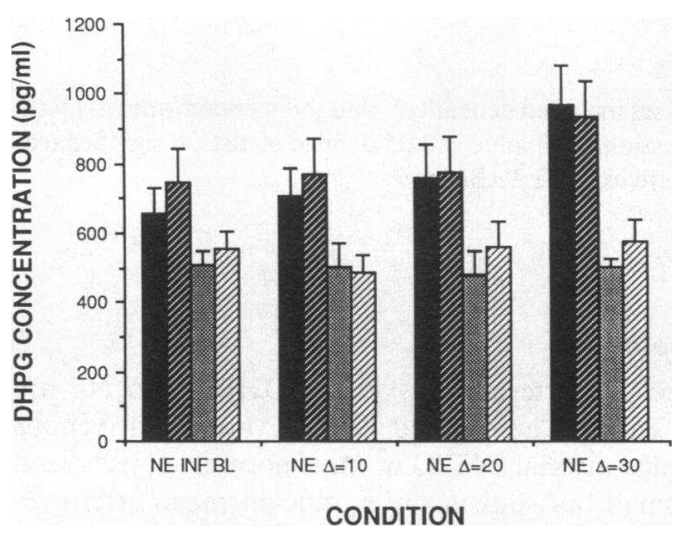

Figure 3. Arterial and venous mean levels of DHPG (DHPGa [ $]$ and DHPGv [æ]) at baseline and during intravenous infusions of NE to achieve mean arterial pressure increments of $10(\Delta=10), 20(\Delta$ $=20)$, and $30(\Delta=30) \mathrm{mm} \mathrm{Hg}$ in subjects with or without pretreatment with DMI. Infusion of NE caused small increases in DHPGa and in DHPGv. These increases were abolished by pretreatment with DMI. DMI/DHPGa (\$); DMI/DHPGv ("). 


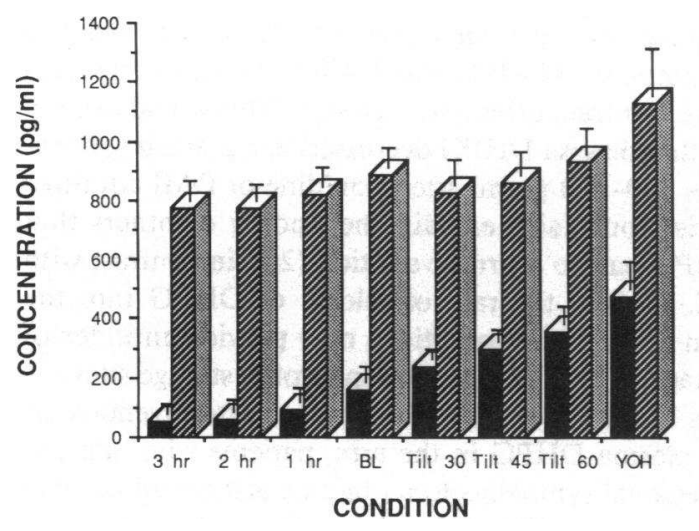

Figure 4. Antecubital venous NE (NEv) (๓) and DHPG (DHPGv) (๗) at baseline (BL), 1, 2, and $3 \mathrm{~h}$ after administration of oral clonidine, at 30,45 , and 60 degrees of tilt from horizontal, and during intravenous administration of $\mathrm{YOH}$. BL values displayed are for subjects before clonidine treatment. As NEv increased, DHPGv increased. When NEv approached zero, DHPGv approached $700-800 \mathrm{pg} / \mathrm{ml}$.

ministration of the alpha-2 adrenoceptor antagonist $\mathrm{YOH}$ and similar absolute decreases, after oral administration of the alpha- 2 adrenoceptor agonist clonidine. The present findings are in agreement with those of others involving measurements of plasma DHPG in humans or laboratory animals (3, 11-19).

Whereas similar changes in NE and DHPG during altered release of endogenous $\mathrm{NE}$ have led others to conclude that simultaneous measurement of plasma DHPG and NE does not offer important advantages over NE alone in the assessment of sympathetic function (19), the results reported here about effects of neuronal uptake blockade lead to a different conclusion. Inhibition of uptake-1 exaggerated NE responses, but abolished DHPG responses to YOH, resulting in dissociation of DHPG responses from NE responses. This suggests that plasma DHPG reflects intraneuronal metabolism of NE, because if increments in plasma DHPG derived extensively from extraneuronal tissues, then during release of endogenous NE, inhibition of uptake-1 would have been expected to increase, not decrease, plasma DHPG.

If plasma NE alone were used to indicate sympathetic activity, then exaggeration of $\mathrm{YOH}$-induced increments in plasma NE during uptake-1 blockade could have led to the inference that DMI enhances the stimulatory effect of $\mathrm{YOH}$

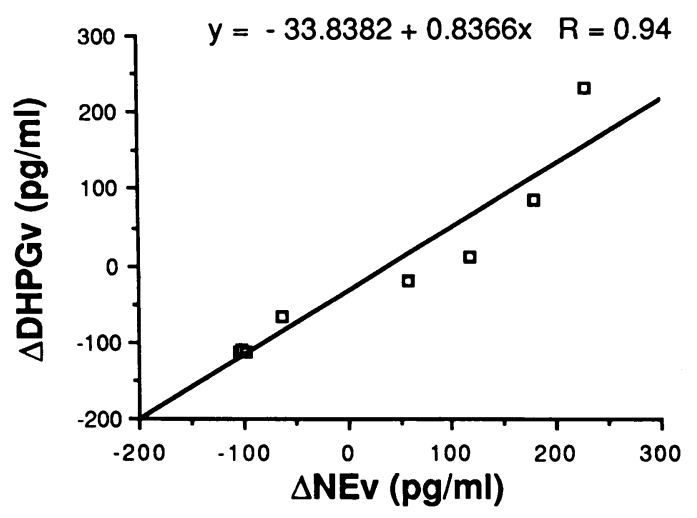

Figure 5. Relationship between the change in mean antecubital venous DHPG $(\triangle D H P G v)$ and in mean antecubital NE $(\triangle N E v)$ during the conditions described for Fig. 4.

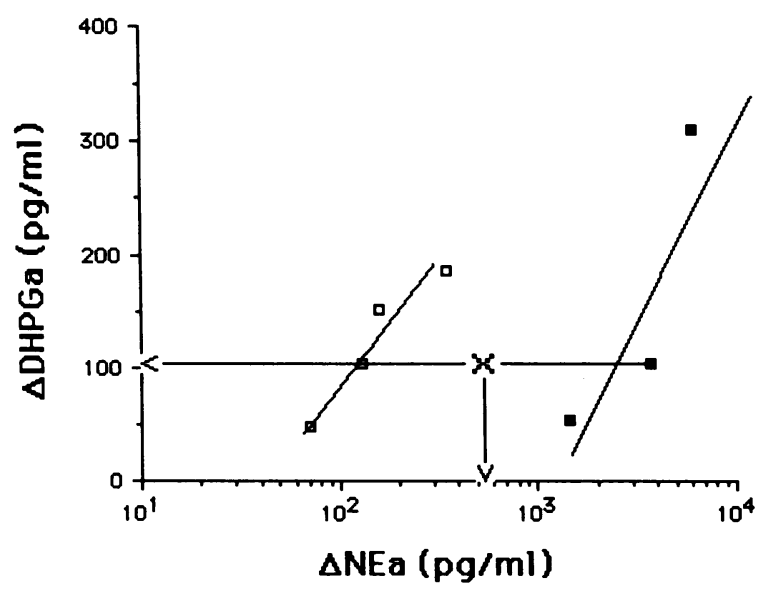

Figure 6. Relationship between the change in mean arterial DHPG $(\triangle \mathrm{DHPGa})$ and in mean arterial $\mathrm{NE}(\triangle \mathrm{NEv})$ during stimuli releasing $\mathrm{NE}$ (open squares) and during NE infusion (closed squares). The mark $\mathrm{X}$ is the location of the geometric mean of the $\triangle \mathrm{NEa}$ concentrations. $\triangle \mathrm{DHPGa}$ increased linearly with the log of $\triangle \mathrm{NEa}$ during release of endogenous $N E$ and during NE infusion. The $\triangle \mathrm{DHPGa} /$ $\triangle \mathrm{NEa}$ relationships were approximately parallel. $\triangle \mathrm{DHPGa}$ values for release of endogenous NE were shifted $\sim 1-\log$ unit to the left of values for $\mathrm{NE}$ infusion.

on central sympathetic outflow; and if plasma DHPG alone were used, then abolition of $\mathrm{YOH}$-induced increments in plasma DHPG during uptake-1 blockade could have led to the inference that DMI diminishes sympathetically mediated transmitter release. Simultaneous measurement of plasma NE and DHPG in this case avoided erroneous inferences about sympathetic function based on behavior of either substance alone.

The same considerations apply to the interpretation of hemodynamic responses to exogenously administered NE during uptake-1 blockade. The shift to the left in the pressor-log arterial NE concentration relationship during uptake-1 blockade could have indicated that uptake-1 blockade affects responsiveness of vascular smooth muscle to exogenously administered NE; however, this would not explain the lack of effect of NE infusion on plasma DHPG during uptake-1 blockade, a finding that is more consistent with increased delivery of exogenously administered NE to postsynaptic alpha-adrenoceptors. Therefore, simultaneous measurement of plasma DHPG and NE provided information about the cause of pressor hyperresponsiveness to exogenously administered NE that was not provided by measurement of plasma NE alone.

The effects of uptake-1 blockade on plasma DHPG responses were similar to previously reported effects of uptake-1 blockade on release of DHPG in isolated vascular beds (1). Uptake-1 blockade has been shown to abolish DHPG release and to enhance NE release during electrical stimulation of tissues preincubated with tracer-labeled NE (20-23). Similarly, inhibition of uptake-1 enhances stimulation-induced release of endogenous NE while decreasing endogenous DHPG release (24). Thus, increments in DHPG release from tissue into incubation medium in laboratory animals and into plasma in humans appear to derive from recapture of NE by sympathetic nerves.

During intravenous administration of NE at physiologically active doses, dose-related increases in plasma DHPG 
were observed. The increments in plasma DHPG were small compared with the NE concentrations, a pattern similar to that reported by lzzo et al. (12). This would be expected from the large concentration gradient between NE in plasma and $\mathrm{NE}$ at neuroeffector junctions, where the drug could be taken up by uptake-1 to provide substrate for DHPG formation. In subjects pretreated with DMI, no change in plasma DHPG was observed during NE administration. Although the NE concentration required to produce the same pressor response was much smaller in subjects pretreated with DMI than in subjects who were not, if plasma DHPG derived substantially from extraneuronal metabolism of NE, then the approximately ninefold increase in arterial NE during NE infusion still would have been expected to increase plasma DHPG $(1,25)$.

The relatively small increments in plasma DHPG during NE infusions in the present study contrast with much larger percent increments in the study of Howes et al. (26), who used tracer-labeled NE. Physiologically active doses of NE, as used in the present study, would be expected to stimulate arterial baroreceptor afferent activity and, because of reflexive sympathoinhibition, to attenuate DHPG responses; however, even using tracer-labeled NE we have failed to confirm the findings of Howes et al. On the basis of results obtained using a similar batch of tracer-labeled NE from the same manufacturer, we have suggested that the large increments in plasma radioactive DHPG reported in the study of Howes et al. may have been caused by contamination of the infusate with DHPG (27).

\section{Source and meaning of plasma DHPG under resting conditions}

One may hypothesize that although increments in DHPG during sympathetic stimulation may reflect intraneuronal production of DHPG, plasma DHPG under resting conditions may reflect extraneuronal production of DHPG from endogenously released or circulating NE. The present results do not exclude this possibility but do question it. If extraneuronal metabolism of endogenously released or circulating NE determined the basal levels of plasma DHPG, then clonidine administration, which caused marked suppression of plasma NE, would have been expected to decrease DHPG to low levels, and NE infusions, which produced large-magnitude increases in plasma NE, would have been expected to produce largemagnitude increases in DHPG. Instead, substantial levels of plasma DHPG were observed after clonidine administration, and DHPG production was more pronounced during stimulation of endogenous NE release than during NE infusion, a finding that can be attributed to DHPG release from sites near neuroeffector junctions. The basal level of DHPG therefore probably did not result from extraneuronal metabolism of endogenously released or circulating NE.

Because of the small proportionate decrease in plasma DHPG after clonidine administration, one would predict that if NE release were suppressed completely, a plasma DHPG concentration of $\sim 700-800 \mathrm{pg} / \mathrm{ml}$ would be observed. In subjects treated with DMI at a dose previously shown to inhibit uptake-1 effectively $(9,10)$, plasma DHPG decreased to a similar concentration range. These results, consistent with those of others about effects of clonidine or uptake-1 blockade on plasma DHPG $(12,14-20)$ suggest that basal release of DHPG depends on a source other than NE recaptured by uptake-1. According to the model in Fig. 1, under conditions where uptake of endogenously released NE is very small, a substantial amount of DHPG could still be produced by leakage of NE from intraneuronal storage sites. This would explain the finding that plasma DHPG decreased to a plateau concentration of $\sim 700-800 \mathrm{pg} / \mathrm{ml}$ after clonidine or DMI administration. This would also explain the finding of others that plasma DHPG has no diurnal variation (28), in contrast with plasma NE. In fact, the rate of release of DHPG into the bloodstream under these conditions may provide an index of the net leakage of NE from the intraneuronal storage sites.

Whereas healthy subjects usually had an arteriovenous increment in plasma DHPG in the arm, patients who had undergone a regional sympathectomy had an arteriovenous decrement in plasma DHPG in the affected limb. This result suggests that release of DHPG into the bloodstream depends on intact sympathetic innervation.

In summary, since blockade of Uptake-1 virtually abolished plasma DHPG responses to YOH or NE infusion, the above findings indicate that increments in plasma DHPG probably reflect intraneuronal production of DHPG from NE recaptured by uptake-1, and since substantial levels of plasma DHPG were observed even when endogenous release of NE was suppressed or when uptake-1 was blocked, plasma DHPG under resting conditions appears to be derived mainly from intraneuronal leakage of NE from storage vesicles into the cytoplasm.

\section{Use of plasma NE and DHPG to estimate NE concentrations at neuroeffector junctions}

We previously have provided evidence in humans that a large concentration gradient for NE operates between the neuroeffector junctions and the plasma (7). By analysis of blood pressure responses to $\mathrm{NE}$ infusions and endogenously released $\mathrm{NE}$, we estimated that for a $20-\mathrm{mm} \mathrm{Hg}$ increment in blood pressure, the concentration of $\mathrm{NE}$ at the neuroeffector junctions would be $\sim 3 \mathrm{nM}(\sim 500 \mathrm{pg} / \mathrm{ml})$. We also determined that NE concentration gradients between the plasma and neuroeffector junctions probably are reciprocal and equal.

Simultaneous measurement of plasma DHPG and NE responses during release of endogenous $\mathrm{NE}$ and $\mathrm{NE}$ infusion provides another means to estimate NE concentrations at neuroeffector junctions (or, more accurately, at uptake-1 sites). Fig. 6 depicts relationships between changes in mean arterial DHPG and changes in mean arterial NE during stimulation of $\mathrm{NE}$ release ( $\mathrm{YOH}$ and three levels of tilt) and during NE infusion (three rates of infusion). For a given increment in the mean arterial level of DHPG, the arterial NE concentration was $\sim 1$ - $\log$ unit smaller for stimulation than for infusion, and the average NE concentration at uptake- 1 sites must have been between the arterial NE concentrations for release and for infusion. Since the present results have implied that increments in plasma DHPG during NE release or NE infusion have the same source, uptake of NE by uptake 1, then given that the concentration gradient between the neuroeffector junctions and the plasma during NE release is the reciprocal of the NE concentration gradient during NE infusion, NE concentrations in the synaptic clefts can be calculated as follows:

During NE infusion, a plasma-cleft concentration gradient, $f_{p-c}$, would relate the increment in the arterial concentration of NE, $[\Delta N E a]_{i n f}$, to the increment in the cleft concentration of NE, $[\Delta N E c]_{i n f}$, and during NE release, a cleftplasma concentration gradient, $f_{c-p}$, would relate $[\Delta N E c]_{r e l}$ to 
$[\Delta N E a]_{r e l}:[\Delta N E a]_{i n f} \times f_{p-c}=[\Delta N E c]_{i n f}$, and $[\Delta N E a]_{r e l}$ $=[\Delta N E c]_{\text {rel }} \times f_{c-p}$.

If increments in plasma DHPG depend on neuronal uptake of NE, regardless of the source of the NE, then for a given increment in plasma DHPG during NE infusion or release of endogenous NE, $[\Delta N E c]_{i n f}=[\Delta N E c]_{r e l}$. Since $f_{c-p}$ and $f_{p-c}$ are assumed to be reciprocal and equal, $[\Delta N E c]^{2}=[\Delta N E a]_{i n f}$ $\times[\Delta N E a]_{r e l}$, and $\log [\Delta N E c]=\left(\log [\Delta N E a]_{\text {inf }}+[\Delta N E a]_{r e l}\right) / 2$.

Thus, the increment in the cleft NE concentration for a given increment in the mean arterial level of DHPG would be half-way on a $\log$ scale between the increments in arterial NE for infusion and stimulation, (approximately at point $X$ in Fig. 6). At an increment of $\sim 100 \mathrm{pg} / \mathrm{ml}$ in arterial DHPG, the estimated increment in cleft NE would be between $\sim 500$ and $600 \mathrm{pg} / \mathrm{ml}$. This value agrees well with values we previously reported in humans and rats $(7,29)$. Since the NE concentration gradient between the plasma and the sites of production of DHPG in the present study was the same as the previously reported NE concentration gradient between the plasma and the neuroeffector junctions, the results also are consistent with intraneuronal production of DHPG. Although a previous study indicated that the cleft-plasma and plasma-cleft concentration gradients probably are reciprocal and equal (29), no experimental data are available to confirm or refute the assumed reciprocal gradients to and from plasma and uptake-1 sites.

\section{DHPG/NE ratio and localization of alterations in NE release or metabolism}

Combined measurement of DHPG and NE at rest and during stimulation can provide clinical information about the release and intraneuronal disposition of the sympathetic transmitter. For instance, patients with pheochromocytoma generally have high plasma NE and slightly increased plasma DHPG, so that the NE/DHPG ratio is high $(14,30)$. This would be expected if the tumor released NE into the bloodstream and the large plasma-cleft concentration gradient resulted in delivery of a small proportion of the circulating NE to neuronal sites of DHPG production. Because basal DHPG production normally far exceeds basal NE release, however, the plasma DHPG/NE ratio itself may not reflect accurately neuronal uptake or metabolism of NE at low rates of NE release.

The $\triangle \mathrm{DHPG} / \triangle \mathrm{NE}$ relationship during alterations in endogenous NE release of during NE infusion would be expected to be shifted to the right in situations where uptake-1 is blocked, such as during treatment with tricyclic antidepressants or administration of cocaine, or MAO is blocked, such as during treatment with a MAO inhibitor for depression. The $\triangle \mathrm{DHPG} / \triangle \mathrm{NE}$ relationship would be expected to be shifted to the left when uptake of cytoplasmic NE into storage vesicles is inhibited, as during administration of reserpine.

\section{Conclusions}

In conclusion, the present results indicate that plasma DHPG is derived from DHPG produced in sympathetic nerve endings; that intraneuronal production of DHPG depends on removal of released NE by uptake-1 and leakage of NE from intraneuronal storage sites, the latter process predominating under basal conditions; that NE concentrations at neuroeffector junctions can be estimated from increments in plasma DHPG and NE during NE infusions and stimulation of endogenous NE release; and that several clinical or pharmacological situations involving altered intraneuronal NE metabolism can be detected by simultaneous measurement of responses of DHPG and NE. Thus, simultaneous measurement of plasma DHPG and NE provides information about sympathetic function that cannot be obtained by measuring either alone.

\section{References}

1. Kopin, I. J. 1985. Catecholamine metabolism: basic aspects and clinical significance. Pharmacol.Rev. 37:333-364.

2. Eisenhofer, G., D. S. Goldstein, R. Stull, H. R. Keiser, T. Sunderland, D. L. Murphy, and I. J. Kopin. 1986. Simultaneous liquid chromatographic determination of 3,4-dihydroxyphenylglycol, catecholamines, and 3,4-dihydroxyphenylalanine in plasma and their responses to inhibition of monoamine oxidase. Clin. Chem. 32:20302033.

3. Izzo, J. L., Jr., and D. Greulich. 1983. Radioenzymatic assay for plasma dihydroxyphenylglycol (DHPG), dihydroxymandelic acid (DOMA), and dihydroxyphenylacetic acid (DOPAC). Life Sci. 33:483-488.

4. Goldstein, D. S., R. McCarty, R. J. Polinsky, and I. J. Kopin. 1983. Relationship between plasma norepinephrine and sympathetic neural activity. Hypertension (Dallas). 5:552-559.

5. Cooper, J. R., F. E. Bloom, and R. H. Roth. 1982. The Biochemical Basis of Neuropharmacology. Oxford University Press, New York. 129-130.

6. Goldstein, D. S., P. D. Levinson, R. Zimlichman, A. Pitterman, R. Stull, and H. R. Keiser. 1985. Clonidine suppression testing in essential hypertension. Ann. Intern. Med. 102:42-48.

7. Goldstein, D. S., R. Zimlichman, R. Stull, H. R. Keiser, and I. J. Kopin. 1986. Estimation of intrasynaptic norepinephrine concentrations in man. Hypertension (Dallas). 8:471-475.

8. Goldstein, D. S., R. F. Bonner, R. Zimlichman, T. P. Zahn, R. O. Cannon, III, D. R. Rosing, R. Stull, and H. R. Keiser. 1986. Indices of sympathetic vascular innervation in sympathectomized patients. J. Auton. Nerv. Syst. 15:309-318.

9. Goldstein, D. S., R Zimlichman, R Stull, J. Folio, P. D. Levinson, H. R. Keiser, and I. J. Kopin. 1985. Measurement of regional neuronal removal of norepinephrine in man. J. Clin. Invest. 76:15-21.

10. Goldstein, D. S., D. Horwitz, H. R. Keiser, R. J. Polinsky, and I. J. Kopin. 1983. Plasma I- $\left[{ }^{3} \mathrm{H}\right]$ norepinephrine, d- $\left[{ }^{14} \mathrm{C}\right]-$ norepinephrine, and d,I-[ $\left.{ }^{3} \mathrm{H}\right]$ isoproterenol kinetics in essential hypertension. J. Clin. Invest. 72:1748-1758.

11. Elsworth, J. D., R. H. Roth, and D. E. Redmond, Jr. 1983. Relative importance of 3-methoxy-4-hydroxyphenylglycol and 3,4-dihydroxyphenylglycol as norepinephrine metabolites in rat, monkey, and humans. J. Neurochem. 41:786-793.

12. Izzo, J. L., Jr., D. A. Thompson, and D. Horwitz. 1985. Plasma dihydroxyphenylglycol (DHPG) in the in-vivo assessment of human neuronal norepinephrine metabolism. Life Sci. 37:1033-1038.

13. Dubocovitch, M. L., and S. Z. Langer. 1976. Influence of the frequency of nerve stimulation on the metabolism of 3H-norepinephrine released from the perfused cat spleen: differences observed during and after the period of stimulation. J. Pharmacol Exp. Ther. 198:83101.

14. Mizukoshi, M., T. Hano, M. Kuchii, I. Nishio, and Y. Masuyama. 1985. Plasma noradrenaline and its deaminated metabolites in essential hypertension and pheochromocytoma. Jpn. Circ. J. 49:1035-1042.

15. Li, P. P., J. J. Warsh, and D. D. Godse. 1986. Further characterization of brain 3,4-dihydroxyphenylethyleneglycol (DHPG) formation: dependence on noradrenergic activity and site of formation. Naunyn-Schmiedeberg Arch Pharmakol. 332:26-33.

16. Li, P. P., J. J. Warsh, D. D. Godse, and M. Guttman. 1985. Studies on the utility of urinary 3,4-dihydroxyphenylethylene-glycol (DHPG) measurement. Prog. Neuro-Psychopharmacol \& Biol. Psychiatry. 9:717-720.

17. Zavadil, A. P., III, R. J. Ross, H. M. Calil, M. Linnoila, P. 
Blombery, D. C. Jimerson, I. J. Kopin, and W. Z. Potter. 1984. The effect of desmethylimipramine on the metabolism of norepinephrine. Life Sci. 35:1061-1068.

18. Robinson, D. S., G. A. Johnson, A. Nies, J. Carcella, T. B. Cooper, D. Albright and D. Howard. 1983. Plasma levels of catecholamines and dihydroxyphenylglycol during antidepressant drug treatment. J. Clin. Psychopharmacol. 3:282-287.

19. Howes, L. G., C. C. Hawksby, and J. L. Reid. 1986. Comparison of plasma 3,4-dihydroxyphenylethylene glycol (DHPG) and norepinephrine levels as indices of sympathetic activity in man. Eur. $J$. Clin. Invest. 16:18-21.

20. Brandoa, F. 1977. Inactivation of norepinephrine in an isolated vein. J. Pharmacol. Exp. Ther. 203:23-29.

21. Brandoa, F., J. G. Monteiro, and W. Osswald. 1978. Differences in the metabolic fate of noradrenaline released by electrical stimulation or by tyramine. Naunyn-Schmiedebergs Arch. Pharmakol. 305:37-40.

22. Endo, T., K. Starke, A. Bangerter, and H. D. Taube. 1977. Presynaptic receptor systems on the noradrenergic neurones of the rabbit pulmonary artery. Naunyn-Schmiedebergs Arch. Pharmakol 296:229-247.

23. Johnston, J. P. 1968. Some observations upon a new inhibitor of monoamine oxidase in brain tissue. Biochem. Pharmacol. 17:1285-1297.

24. Mishima, S., H. Miyahara, and H. Suzuki. 1984. Transmitter release modulated by alpha-adrenoceptor antagonists in the rabbit mesenteric artery: a comparison between noradrenaline outflow and electrical activity. Br. J. Pharmacol. 83:537-547.

25. Maas, J. W., and D. H. Landis. 1971. Metabolism of circulating norepinephrine in human subjects. J. Pharmacol. Exp. Ther. 177:600-612.

26. Howes, L. G., A. MacGilchrist, C. Hawksby, D. Sumner, and J. L. Reid. 1986. An improved approach for the determination of plasma [3H]noradrenaline kinetics using high-performance liquid chromatography. Clin. Sci. 71:211-215.

27. Eisenhofer, G., D. S. Goldstein, R. Stull, T. G. Ropchak, H. R. Keiser, and I. J. Kopin. 1987. Dihydroxyphenylglycol and dihydroxymandelic acid during intravenous infusions of noradrenaline. Clin. Sci. 83:123-127.

28. Dennis, T., C. Benkelfar, Y. Touitou, A. Auzeby, M.-F. Poirier, B. Scatton, and H. Loo. 1986. Lack of circadian rhythm in plasma levels of 3,4-dihydroxyphenylethyleneglycol in healthy human subjects. Psychopharmacology. 90:471-474.

29. Kopin, I. J., Z. Zukowska-Grojec, M. A. Bayorh, and D. S. Goldstein. 1984. Estimation of intrasynaptic norepinephrine concentrations at vascular neuroeffector junctions in vivo. Naunyn-Schmiedebergs Arch. Pharmakol. 325:298-305.

30. Brown, M. 1984. Simultaneous assay of noradrenaline and its deaminated metabolite dihydroxphenylglycol, in plasma: a simplified approach to the exclusion of phenochromocytoma in patients with borderline elevation of plasma noradrenaline concentration. Eur. J. Clin. Invest. 14:67-72. 